\title{
New Models for Home Health Care under Uncertainty with Consideration of the Coordinated Development of Economy and Environment
}

\author{
Yan Cui $\mathbb{D}^{1,2}$, Lijun Zhang $\left(\mathbb{D},{ }^{3}\right.$ Qiuye Gao ${ }^{1},{ }^{3}$ Bohao Li $\left(\mathbb{D},{ }^{4}\right.$ and Yumei Hou ${ }^{1}{ }^{1}$ \\ ${ }^{1}$ School of Economics and Management, Yanshan University, Qinhuangdao, Hebei, China \\ ${ }^{2}$ LiRen College, Yanshan University, Qinhuangdao, Hebei, China \\ ${ }^{3}$ Information Management Department, The First Hospital of Qinhuangdao, Qinhuangdao, Hebei, China \\ ${ }^{4}$ School of Gemmology, China University of Geosciences(Beijing), Beijing, China \\ Correspondence should be addressed to Yumei Hou; hym@ysu.edu.cn
}

Received 27 November 2020; Revised 17 December 2020; Accepted 1 January 2021; Published 28 January 2021

Academic Editor: Wei Zhang

Copyright ( $\odot 2021$ Yan Cui et al. This is an open access article distributed under the Creative Commons Attribution License, which permits unrestricted use, distribution, and reproduction in any medium, provided the original work is properly cited.

\begin{abstract}
With the aggravation of population aging, home health care (HHC) services are paid more and more attention by the elderly. Previous studies aim at improving service quality and reducing cost, ignoring the coordinated and sustainable development of the economy and environment. From the perspective of sustainable development, this paper first establishes a linear optimization (LO) model considering transportation, time, and carbon emission costs. However, the uncertainty of service demand is a very difficult problem for HHC research. Most of the previous studies only consider the deterministic model, which has difficulty dealing with the uncertain situation. Therefore, a robust optimization (RO) model is proposed to resist uncertain disturbances by introducing a robust uncertain set response. The experimental results show that the increase of low-carbon transition cost only increases the total cost of the LO model but has a significant positive impact on the RO model. With the increase of uncertainty, the robust model will pay the cost of robustness, but it can obtain a higher service level (93.20\% to 93.38\%). In addition, when the carbon tax increases, the total transportation cost does not increase but decreases, thus obtaining environmental benefits. When the carbon tax increases by $25 \%$, the average total cost of using the RO model is reduced by $8.274 \%$. The research results of this paper can provide enlightenment and reference for the low-carbon transformation of HHC enterprises.
\end{abstract}

\section{Introduction}

The aging of the population has become a major problem restricting the development of the 21st century. As a new type of service, home health care is quietly changing people's lives. In the past, researches only focused on how to improve social welfare but ignored the coordinated development of environmental ecology. The concept of HHC first appeared in 1945 [1]. Later, with the passing of the times and the progress of technology, it gradually entered the life of ordinary residents [2]. HHC provides door-to-door services such as health care for people who are dependent and implements different types of care according to the needs of specific people [3]. Fikar and Hirsch (2017) divide the HHC service into three steps, which are collecting information, formulating resource allocation, and path planning [4]. In the context of the two eras, the increasing aging of the population, and the substantial improvement in material life, the demand for HHC services is rapidly increasing. In recent years, the healthcare industry has become one of the largest economic sectors in Europe and North America [5]. Cho et al. (2018) found that the effective implementation of HHC work is of great help to improve the quality of people' life [6]. At present, HHC can be regarded as a supplementary form of public health under the auspices of the government. Through the door-to-door service, allowing the demanders to receive treatment at home can alleviate the resource shortage caused by the limited public health resources [7]. 
While people enjoy the convenient services brought by $\mathrm{HHC}$, the increasing energy consumption and environmental pollution problems are becoming increasingly prominent. In recent years, environmental problems have become increasingly prominent, and greenhouse gas emissions have become more stringent. In the path planning problem, we must not only consider economic costs, but environmental costs are also an important factor that affects path selection. How to find a balance between economy and environment is worth exploring. At the strategic level, Wang et al. (2018) found that cooperative strategies can minimize carbon emissions and achieve green vehicle path planning [8]. Yu et al. (2019) proposed an improved branching and pricing algorithm to solve the routing problem of heterogeneous green fleet vehicles with time windows [9]. Bettinelli et al. (2019) studied the problem of urban double-layer logistics path planning and found that it is of positive significance to coordinate the arrival of facility vehicles and nursing staff with corresponding time constraints [10]. At the same time, HHC is also facing general problems and emerging challenges today. The most important goal of an $\mathrm{HHC}$ service company is to meet the needs of the demanders in a timely manner. During this process, two main operational problems are often encountered. One is the delay in service to the demanding personnel, and the other is the travel of the medical team routing problem. Of these two, the issue of time delay is particularly important, which not only punishes service providers but also may lead to more serious consequences for improper treatment of demanders. Therefore, while the operating platform is pursuing profits, how to effectively plan limited resources is the primary factor that needs to be considered for HHC [11]. It can be seen that the path planning of family medical and nursing services has become a key issue for service providers to reduce operating costs and improve service quality. In a fiercely competitive market environment, HHC companies of the traditional chain will face a severe impact from Internet informatization, especially today, with the growing development of technology and big data. HHC companies that operate on network platforms may become the main subject of industry $[12,13]$.

For the HHC service path planning problem, many scholars have used different theoretical methods to study operation options of nursing station selection and vehicle route allocation. In general, those two well-known operation options play a key role [14]. Shore (1999) explored a new world of health care by creating a health policy implementation plan [15]. Matteo (2000) constructed an empirical model of mixed determinants based on data from Canada's total health expenditure during the 1975-1996 decade and the usage of hospitals, doctors, and drugs [16].

Current research results on path planning can be divided into the following two categories, deterministic path planning models and uncertain path planning models. Moussavi et al. (2017) outlined how to carry out planning activities in the HHC structure, especially the route and arrangement of nursing staff, and pointed out that patients are the core of the decision-making system [14]. In terms of reality, at the same time, it is considered that the route and arrangement of nursing staff are the basic problems in transportation planning during the path planning process. In the path planning stage, under normal circumstances, decision makers tend to consider relevant parameters as deterministic values [17]. Some scholars have conducted in-depth research on green logistics $[18,19]$. However, in the actual market operating environment, even with the support of advanced network technologies such as big data and the Internet, some parameters are still unpredictable, such as specific demand, key parameters such as the customer's waiting time; uncontrollable factors, such as road conditions, weather conditions, and even emergency emergencies. Therefore, the deterministic path optimization model has difficulty in dealing with these unknown factors. The application of robust optimization can be used to deal with uncertainty [20, 21]. Since the robust optimization method does not depend on the characteristics of the probability distribution of data, it has unique advantages in solving uncertain problems [22]. In the path planning problem, scholars use robust optimization proposed by Ben-Tal et al. (2002) to deal with uncertainty [23]. In the research field of uncertain decisionmaking problems, compared with stochastic programming, the robust optimization model is more flexible. The principle of robust optimization is that the uncertainty of parameters is irregular, which is the biggest difference between robust optimization and stochastic programming. For robust optimization, there is no need to restrict the membership rules of parameters, which avoids the controversy. Thus, the robust optimization is more general in describing randomness, and the model is more compatible. In general, robust optimization can adjust the size of the fluctuation range space of parameters to meet the needs of different decision makers for different risk attitudes.

Robust optimization focuses on constructing uncertain sets and describing uncertain problems, such as Box sets, Ellipsoids, or Polyhedron, and measuring the risk through changes in uncertain sets $[24,25]$. One advantage of robust optimization is that as long as the appropriate uncertainty set is designed, the consistency between the robust correspondence model and the original problem model can be guaranteed [26]. The research of robust optimization was further developed. Montemanni (2005) proposed a branch and bound algorithm for the robust spanning tree problem [27]. Sarac et al. (2006) invented a branch-price algorithm to solve and optimize the problem of abnormal airline routes [28]. Subsequently, Zhang et al. (2007) established a multigranular robust routing model in a mesh network and proposed a load balancing robust routing scheme [29]. Huang and Wang (2009) used the optimized genetic algorithm to solve the robust optimal network solution of multiobjective optimization and proved the convergence of the algorithm through numerical experiments [30]. Some scholars have studied the home health care routing problem through an intelligent algorithm and verified the feasibility of the paper model through practical cases [31-34]. In addition, some scholars have studied uncertain decisionmaking problems and multicriteria decision-making problems through robust optimization [35-38]. Therefore, based on the above characteristics, the robust optimization method 
has extensive research value in the theoretical and practical fields. To the best of our knowledge, very few scholars currently apply robust optimization theories and methods to the path planning of HHC, and this article is to supplement this, so it has considerable research value. The main contributions of this article are as follows:

(i) Taking carbon emission cost as an important reference factor for path planning

(ii) According to the actual situation, build a LO model of HHC services

(iii) Introduce uncertain parameters to further expand the LO model into three RO models and construct different security level models through three uncertain sets

(iv) In the case of numerical case analysis, use real case data for analysis and provide routing planning solutions for path planning decisions of actual HHC services companies

It can be seen that the innovation of robust optimization theory is of great significance and the application field is extremely broad. At present, most HHC related studies only consider deterministic models and less uncertainty. Therefore, the decisions given in these solutions have limited applicability in practical situations [39, 40]. In addition, even though there are some researches on uncertainties in family medical services, they have not intervened from the perspective of robust optimization. Therefore, from the perspective of robust optimization, this paper studies the path planning of HHC under uncertain parameters. According to the literature review, this article will be one of the first important studies to apply robust optimization theory to HHC path planning. Although the literature has elaborated on the application of robust optimization theory, the model is basically a single set of uncertain sets. As an improvement, this paper will propose and construct a robust optimization under multiple sets of uncertain sets, so the model has a deeper and richer research level.

The rest of this article is organized as follows. Section 2 is the basic model. Section 3 describes the HHC path problem and constructs the LO model to solve the two-level path planning problem.Section 4 transforms the LO model into three RO models.Section 5 conducts numerical experiments and analyzes the results.Section 5 summarizes the research work of this article and summarizes and proposes future research directions.

\section{Basic Model}

This paper studies the problem of two-level routing planning for the HHC problem under uncertain environment (Figure 1). In this problem, two types of sites are considered: home care and nursing stations and demand communities. On the one hand, the home care station deals with the order demand from the demand community. The number of nursing stations needs to be considered comprehensively to minimize operation and management costs. On the other hand, considering the cost minimization and demand

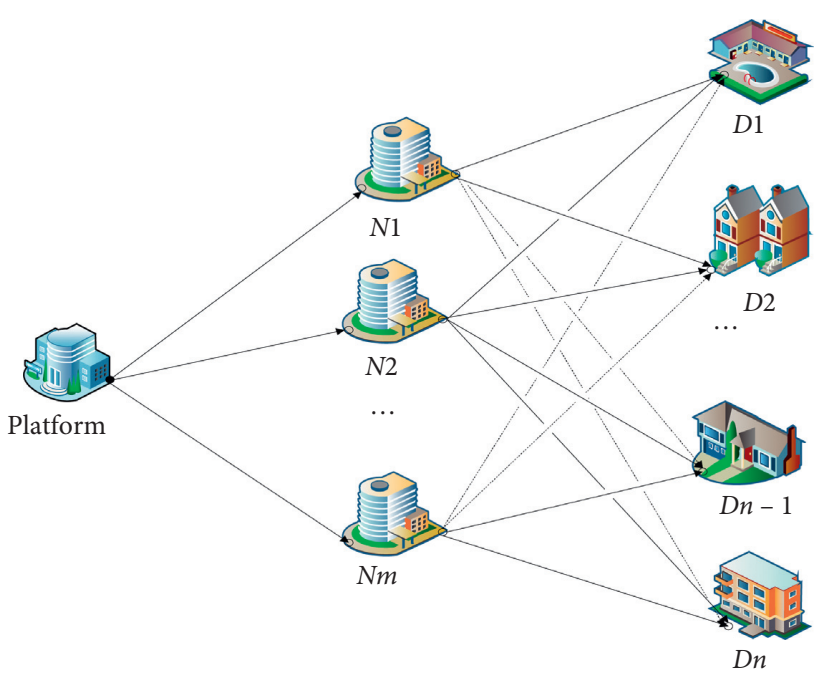

FIGURE 1: Schematic diagram of routing planning for HHC.

responsiveness, optimize the driving routing of service personnel. On the basis of meeting the community needs to the maximum extent, the driving cost of vehicles can be reduced through reasonable planning routing. In our study, cost types are considered including fixed cost, vehicle cost, time cost, and transportation cost [41]. This paper studies the optimal routing planning of $M$ HHC stations and $N$ demand communities and establishes an optimization model of HHC. The purpose is to meet the needs of customers to the maximum extent and minimize the total cost under the constraints of nursing station, time, and road condition.

2.1. Symbolic Variable Description. The parameters and variables used in this model are described in Table 1.

2.2. Basic Assumptions. The following assumptions for the above routing planning problems are presented:

(i) The problem of routing planning is to provide medical and nursing services from one platform nursing station to multiple communities in need

(ii) The needs of all communities must be met

(iii) At least one nursing station provides nursing services at each demand point

(iv) The vehicles used are of the same model with the same fuel consumption and load capacity

(v) Both the time window for the geographic location and needs of the community are known

(vi) The average speed of vehicles in different regions is different, which depends on the road conditions and time period in the region

2.3. Cost Analysis. This study reflects the actual situation of the medical care routing planning process under the operation of a sharing platform. The specific cost includes the fixed cost of the medical care station, transportation cost, vehicle carbon emission cost, and time cost. 
TABle 1: Parameters and variables.

\begin{tabular}{|c|c|c|}
\hline Type & Symbol & Description \\
\hline \multirow{2}{*}{ Decision variables } & $x_{i}$ & $x_{i} \in[0,1]$, continuous variable. If $x_{i} \neq 0$, select $i$ \\
\hline & $y_{i j}$ & $y_{i j} \in[0,1]$, continuous variable. If $y_{i j} \neq 0$, select $i j$ \\
\hline \multirow{12}{*}{ Basic parameters } & $D_{j}$ & The demand of HHC services \\
\hline & $c_{f}$ & Fixed cost of nursing station \\
\hline & $H_{i}^{\mathrm{Max}}$ & Maximum resource allocation of nursing station $i$ \\
\hline & $h_{v}$ & Maximum load of HHC vehicle \\
\hline & $c_{v}$ & Unit vehicle transportation fuel consumption cost \\
\hline & $E_{c}$ & Unit fuel consumption of nursing vehicles \\
\hline & $c_{t}$ & Penalty cost per unit delay \\
\hline & $c_{e}$ & Carbon tax \\
\hline & $d_{i j}$ & Distance between demand community and nursing station \\
\hline & $\bar{v}_{i}$ & Average speed of the area where nursing station $i$ is located \\
\hline & $t$ & Arrival time of benchmark service personnel \\
\hline & $T_{t}^{\mathrm{Max}}$ & Maximum arrival time \\
\hline \multirow{2}{*}{ Indicating parameters } & $I$ & Nursing station set, consisting of $i$ \\
\hline & $J$ & Demand community aggregation, consisting of $j$ \\
\hline
\end{tabular}

2.3.1. Fixed Cost. Considering the fixed cost, the fixed cost is the infrastructure investment cost, including the loss cost of office equipment, the basic water and electricity cost of the nursing station, and the salary of management. The fixed cost of agricultural sharing platform operation is calculated as follows:

$$
C_{f}=\sum_{i=1}^{m} c_{f}\left\lceil x_{i}\right\rceil, \quad x_{i} \in[0,1],
$$

where, $\left\lceil x_{i}\right\rceil$ means to round up the value, for example, if $x_{i}=0.1$, then round up to $1 ; x_{i}=1.1$ round up to 2 . The actual meaning is that once the warehouse is selected, the fixed cost will exist.

2.3.2. Vehicle Transportation Cost. In order to ensure the accurate arrival of home care personnel, the transportation cost is as follows:

$$
C_{v}=c_{v} \sum_{i=1}^{m} \sum_{j=1}^{n}\left(\frac{D_{j}}{h_{v}}\right) y_{i j} d_{i j} .
$$

2.3.3. Cost of Waiting Time. The cost of waiting time is the reward (or punishment) brought by the advance (or delay) of time, and the specific form is as follows:

$$
C_{t}=c_{t} \sum_{i=1}^{m} \sum_{j=1}^{n}\left\lceil y_{i j}\right\rceil\left(\frac{d_{i j}}{\bar{v}_{i}}-t\right) \text {, }
$$

among them, $c_{t}$ is the unit reward (or punishment) cost related to time, which is the time for a basic nursing home visit, $\bar{v}$ the average speed of vehicles in the area of home care station [42].

2.3.4. Vehicle Carbon Cost. Due to the government's environmental protection requirements, a certain amount of carbon emission tax should be levied on motor vehicles [5]. The specific costs are as follows:

$$
C_{c}=c_{c} \sum_{i=1}^{m} \sum_{j=1}^{n} e d_{i j} E_{c} D_{j} y_{i j} .
$$

2.4. Basic Low-Carbon Linear Optimization Model. To sum up, this paper constructs a low-carbon linear optimization (LO) model [43], which aims to minimize the total cost on the basis of meeting customer needs in the maximum extent. The specific model is as follows:

(Low-carbon LO model)

$$
\begin{gathered}
\min C_{f}+C_{v}+C_{t}+C_{c}, \\
\text { s.t. } \sum_{i=1}^{m} y_{i j}=1, \quad \forall j \in J, \\
\sum_{j=1}^{n} D_{j} y_{i j} \leq H_{i}^{\mathrm{Max}}, \quad \forall i \in I, \\
\left\lceil y_{i j}\right\rceil \cdot\left(\frac{d_{i j}}{\bar{v}_{j}}\right) \leq T_{t}^{\mathrm{Max}}, \quad \forall i \in I, \forall j \in J, \\
C_{c}\left(x_{i}, D_{j}\right) \leq E_{c}^{\mathrm{Max}}, \quad \forall i \in I, \forall j \in J, \\
0 \leq y_{i j} \leq 1, \quad \forall i \in I, \forall j \in J, \\
0 \leq x_{i} \leq 1, \quad \forall i \in I .
\end{gathered}
$$

The specific objective functions and constraints of the basic LO model are as follows. The objective (5) is the synthesis of the above cost analysis, including fixed cost, transportation cost, vehicle carbon emission cost, and time cost. Constraint (6) represents that all HHC services under the platform of the region are provided by the nursing station, without any other external diversion, and the needs of any community under the platform must be met. Constraint (7) the ability of nursing service provided by nursing 
station was less than its maximum ability and constraint (8) is a time constraint, that is, the time consumed by any routing is less than its maximum travel period. Constraint (9) is that the cumulative carbon emission is less than the maximum limit. Constraint (10) means that the resource allocation proportion on any routing is not less than 0 and not more than 1 ; when $y i j=0$, it means that this line is not selected; when $y i j=1$, it means that all the needs of the community are supplied by $i$. Constraint 11 ) indicates that the resource allocation variable of the nursing station is a continuous fractional variable.

\section{RO Models}

In reality, due to the complexity of the external market environment, it is very difficult to obtain the accurate value or probability distribution of key parameters, especially for demand [44]. This leads to the low feasibility or even nonexistence of the ideal model in the real society; that is to say, the robustness of the routing planning scheme of the basic model is low under the uncertain conditions. Therefore, the research of robust optimization theory is more attractive. In this section, using the theory of robust optimization, the above-mentioned deterministic model is transformed into a robust optimization model, so that the uncertain parameters change in an uncertain set, so that the probability distribution can also be carried out to the research of routing planning with the independence of the model. Robust optimization is one of the main methods to achieve the goals [45]. The greater the volatility of customer demand, the greater the uncontrollability. Therefore, it is defined as a random demand parameter $\widetilde{D}_{j}=D_{j}^{0}+\widehat{D}_{j}, D_{j}^{0}$ as nominal demand, $\widehat{D}_{j}=\varepsilon D_{j}^{0}$ as demand fluctuation, and $\varepsilon$ as disturbance proportion. On this basis, the RO models of Box, Polyhedron, and Ellipsoid set are established [43]. In order to facilitate the description, this paper divides the cost into two, one is nominal deterministic item $C_{D}\left(D_{j}^{0}\right)=$ $C_{v}\left(D_{j}^{0}\right)+C_{t}\left(D_{j}^{0}\right)+C_{c}\left(D_{j}^{0}\right)$, the other is $C_{S}\left(\widehat{D}_{j}\right)=C_{v}\left(\widehat{D}_{j}\right)+$ $C_{t}\left(\widehat{D}_{j}\right)+C_{c}\left(\widehat{D}_{j}\right)$, which is affected by uncertain parameters.

3.1. Box-RO Model. In the Box-RO model, the uncertainty requirement is $\widetilde{D}_{j}$, and the uncertainty set is Box [46]. According to the robust optimization theory, the robust equivalent model is (12), $U^{B}=U_{\infty}=\left\{\varepsilon:\|\varepsilon\|_{\infty} \leq \Psi\right\}=$ $\left\{\varepsilon\right.$ : $\left.\left|\varepsilon_{j}\right| \leq \Psi\right\}$ and $\Psi$ is the uncertain level parameter (i.e., safety parameter, SP). SP represents that at most $\Psi$ parameters deviate from nominal value.

Theorem 1. According to the relevant theory of robust optimization, the equivalent robust correspondence of $\{\max$ $\left.c^{T} x \mid A x \leq B, l \leq x \leq u\right\}$ is $\sum_{j} a_{i j} x_{j}+\max _{\xi \in U} \sum_{j} \widehat{a}_{i j} x_{j} \xi_{i j} \leq B$. The resulting Box-RO model is as follows:

(Box-RO model) $\min Z_{B}$

$$
\begin{aligned}
& C_{f}+C_{D}\left(D_{j}^{0}\right)+\psi\left[C_{S}\left(\widehat{D}_{j}\right)\right] \leq Z_{B} \\
& \sum_{i=1}^{m} y_{i j}=1, \quad \forall j \in J \\
& \sum_{j=1}^{n} D_{j}^{0} y_{i j}+\psi \sum_{j=1}^{n} \widehat{D}_{j} y_{i j} \leq H_{i}^{\mathrm{Max}}, \quad \forall i \in I
\end{aligned}
$$

s.t.

$$
\begin{aligned}
& \left\lceil y_{i j}\right\rceil \cdot\left(\frac{d_{i j}}{\bar{v}_{j}}\right) \leq T_{t}^{\mathrm{Max}}, \quad \forall i \in I, \forall j \in J \\
& C_{v}\left(x_{i}, D_{j}\right)+\psi\left[C_{S}^{\prime}\left(\widehat{D}_{j}\right)\right] \leq E_{e}^{\mathrm{Max}}, \quad \forall i \in I, \forall j \in J \\
& 0 \leq y_{i j} \leq 1, \quad \forall i \in I, \forall j \in J \\
& 0 \leq x_{i} \leq 1, \quad \forall i \in I .
\end{aligned}
$$

Proof. General Linear Programming (LP) problem is $\left\{\max c^{T} x \mid A x \leq B, l \leq x \leq u\right\}$. Under uncertain conditions, the uncertain Linear Programming (LP) problem can be expressed as follows:

$$
\left\{\min _{x}\left\{c^{T} x+d: A x \leq B\right\}\right\}_{(c, d, A, B) \in U}
$$

Among them, the cost function is $c^{T} x+d$, the basic constraint is $A x \leq B$, and the support set is $U$. Considering $i^{\text {th }}$ row of matrix $A$, assume that element $\widehat{a}_{i j}$ in $A$ is uncertain, then define that $\tilde{a}_{i j}=a_{i j}+\widehat{a}_{i j} \xi_{i j}$, where $\tilde{a}_{i j}$ is true value of parameter, $a_{i j}$ is nominal value while $\widehat{a}_{i j}$ is fluctuation of parameter, $\xi_{i j}$ is factor ( $\xi \in U, U$ is the uncertainty set), $\xi$ can take any possible value in $U$. So, (5) can be represented as (6). Then, uncertain sets and their corresponding robust equivalences are as follows:

$$
\sum_{j} a_{i j} x_{j}+\max _{\xi \in U} \sum_{j} \hat{a}_{i j} x_{j} \xi_{i j} \leq B .
$$

And (6) is equivalent to $\sum_{\mathscr{g}} a_{i j} x_{j}+\Psi \sum_{\mathscr{g}} \widehat{a}_{i j}\left|x_{j}\right| \leq B$. Set $\mathbb{P}_{\infty}=\left[\mathscr{J}_{L \times L} ; \mathbb{O}_{1 \times L}\right], \mathscr{P}_{\infty}=\left[\mathcal{O}_{L \times 1} ; \Psi\right] \ldots_{\infty}=\left\{\left[\theta_{L \times 1} ; t\right]:\|\theta\|_{\infty}\right.$ $=t\}$, where $L$ is the number of uncertain parameters. Therefore, the inner layer maximization in (6) can be rephrased as $\max _{\xi \in U^{B}}\left\{\sum_{j} \widehat{a}_{i j} x_{j} \xi_{i j}: \mathbb{P}_{\infty} \xi+\mathscr{P}_{\infty} \in \mathbb{K}_{\infty} \Psi\right\}$. Define the dual variable as $w_{i}, \lambda_{i}$, according to dual cone theory $\mathbb{K}_{\infty}^{*}=\left\{\left[\theta_{L \times 1} ; t\right]:\|\theta\|_{l} \leq t\right\}$, then, $\min _{w, \lambda}\left\{\Psi \lambda_{i}: w_{i j}=\widehat{a}_{i j}\right.$ 
$\left.x_{j}, \forall j, \sum_{\mathcal{g}}\left|w_{i j}\right| \leq \lambda_{i}\right\}$, and $\min _{w, \lambda}\left\{\Psi \sum_{\mathcal{g}}\left|w_{i j}\right|: w_{i j} \leq \widehat{a}_{i j} x_{j}, \forall_{j}\right\}$ are equal. Thus, it can be reformed as $\Psi \sum_{\mathcal{g}} \widehat{a}_{i j} x_{j}$. So, Theorem 1 is proved.

3.2. Polyhedron-RO Model. If the uncertainty set of each node is Polyhedron, and the Polyhedron set is defined by $l_{1}$ norm: $U^{P}=U_{1}=\left\{\varsigma:\|\varepsilon\|_{1} \leq \Gamma\right\}=\left\{\varepsilon \cdot \sum\left|\varepsilon_{j}\right| \leq \Gamma_{j}\right\}$, where $\Gamma$ is the uncertain horizontal parameter. The Polyhedron-RO model is given in the following theorem, where $\Gamma$ represents the SP of uncertain demand [47-50].

Theorem 2. The equivalent robust correspondence of $\left\{\max c^{T} x \mid A x \leq B, l \leq x \leq u\right\}$ is $\sum_{j} a_{i j} x_{j}+\Gamma_{i} p_{i} \leq B_{i}, p_{i} \geq \widehat{a}_{i j}\left|x_{j}\right|$. The resulting Polyhedron- $R O$ model is as follows:

(Polyhedron-RO model)

$\min Z_{p}$

$$
\begin{aligned}
& C_{f}+C_{D}\left(D_{j}^{0}\right)+\Gamma\left[C_{S}\left(\widehat{D}_{j}\right)\right] \leq Z_{p} \\
& \sum_{i=1}^{m} y_{i j}=1, \quad \forall j \in J \\
& \sum_{j=1}^{n} D_{j}^{0} y_{i j}+\Gamma^{\prime} C_{S}\left(\widehat{D}_{j}\right) \leq H_{i}^{w}, \quad \forall i \in I
\end{aligned}
$$

s.t.

$$
\begin{aligned}
& \left\lceil y_{i j}\right\rceil \cdot\left(\frac{d_{i j}}{\bar{v}_{j}}\right) \leq T_{t}^{\mathrm{Max}}, \quad \forall i \in I, \forall j \in J \\
& C_{v}\left(x_{i}, D_{j}\right)+\Gamma^{\prime}\left[C_{S}^{\prime}\left(\widehat{D}_{j}\right)\right] \leq E_{e}^{\mathrm{Max}}, \quad \forall i \in I, \forall j \in J \\
& 0 \leq y_{i j} \leq 1, \quad \forall i \in I, \forall j \in J \\
& 0 \leq x_{i} \leq 1, \quad \forall i \in I .
\end{aligned}
$$

Proof. Constraint (16) is equivalent to constraint (14).

$$
\sum_{j} a_{i j} x_{j}+\Gamma_{i} p_{i} \leq B_{i}, \quad p_{i} \geq \widehat{a}_{i j}\left|x_{j}\right| .
$$

Defining that $\mathbb{P}_{1}=\left[\mathscr{J}_{L \times L} ; \mathbb{O}_{1 \times L}\right], \mathscr{P}_{1}=\left[\mathscr{O}_{L \times 1} ; \Gamma\right] \ldots 1=$ $\left\{\left[\theta_{L \times 1} ; t\right]:\|\theta\|_{1}=t\right\}$, where $L$ is the number of uncertain parameters. Therefore, the problem of inner layer maximization in (14) can be rephrased as $\max _{\xi \in U^{P}}\left\{\sum_{\mathscr{f}}\right.$ $\left.\widehat{a}_{i j} x_{j} \xi_{i j}: \mathbb{P}_{1} \xi+\mathscr{P}_{1} \in \mathbb{K}_{1} \Gamma\right\}$. Define the dual variable as $w_{i}, \lambda_{i}$, according to dual cone theory $\mathbb{K}_{1}^{*}=\left\{\left[\theta_{L \times 1} ; \mathscr{T}\right]:\|\theta\|_{l} \leq \mathscr{T}\right\}$.

$$
\begin{aligned}
& \max _{\xi \in U^{P}}\left\{\sum_{\mathcal{J}} \widehat{a}_{i j} x_{j} \xi_{i j}: \mathbb{P}_{1} \xi+\mathscr{P}_{1} \in K_{1}\right\} \\
& =\min _{\omega, \lambda}\left\{\Gamma \lambda_{i}: \omega_{i j}=\widehat{a}_{i j} x_{j}\left\|\omega_{i}\right\|_{\infty} \leq \lambda_{i}\right\} \\
& =\min _{\omega}\left\{\Gamma \max _{\mathcal{J}}\left|\omega_{i j}\right|: \omega_{i}=\widehat{a}_{i j} x_{j}\right\} \\
& =\Gamma \mathscr{P}_{i}, \quad \mathscr{P}_{i} \geq \widehat{a}_{i j}\left|x_{j}\right| .
\end{aligned}
$$

In summary, Theorem 2 can be proved.
3.3. Ellipsoid-RO Model. In the Ellipsoid-RO model, the uncertain parameters are defined according to $l_{2}$ norms, $U^{E}=U_{2}=\left\{\varsigma:\|\varepsilon\|_{2} \leq \Omega\right\}=\left\{\varepsilon \sqrt{\sum_{J} \varepsilon_{j}^{2}} \leq \Omega\right\}$, where $\Omega$ is the adjustable uncertain parameter (safety parameter) and the ball diameter of the uncertain set. The Ellipsoid uncertainty set of $\widetilde{D}_{j}$ is $U^{E}=\left\{\widetilde{D} \in R, \sum_{j=1}^{n}\left[\left(\widetilde{D}_{j}-D_{j}^{0}\right) / \widehat{D}_{j}\right]^{2} \leq \Omega^{2}\right\}$. Since the model is a nonlinear constraint problem, set $U^{E}$ is equivalent to the following form: $U^{E}=\left\{\widetilde{D} \in R,\left(\widetilde{D}_{j}-\right.\right.$ $\left.\left.D_{j}\right)^{T} C^{-1}\left(\widetilde{D}_{j}-D_{j}\right) \leq \Omega^{2}\right\}$, where $\mathrm{C}$ is an n-order diagonal matrix with element (nonzero) of $\widehat{D}_{j}^{2}$, then $C_{11}+C_{D}\left(D_{j}^{0}\right)+$ $\Omega_{1} \sqrt{\sum_{j=1}^{n} \widehat{D}_{j}^{2}\left(\sum_{i=1}^{m} \sum_{j=1}^{n} y_{i j} c_{e}\right)^{2}} \leq Z_{E}$. Let $r_{i}=\sum_{i=1}^{m} \sum_{j=1}^{n} y_{i j} c_{e}$, $P=\sqrt{\sum_{j=1}^{n} \widehat{D}_{j}^{2} r_{i j}^{2}}$, then the above formula is transformed to $C_{11}+C_{D}\left(D_{j}^{0}\right)+\Omega_{1} P \leq Z_{E}$. Since the goal is to minimize the total cost, then add relaxation constraint $P \geq \sqrt{\sum_{j=1}^{n} \widehat{D}_{j}^{2} r_{i j}^{2}}, r_{i}$ $\geq \sum_{i=1}^{m} \sum_{j=1}^{n} y_{i j} c_{e}$.

Theorem 3. The equivalent robust correspondence of $\left\{\max c^{T} x \mid A x \leq B, l \leq x \leq u\right\} \quad i s a_{i}^{T} X+\Omega_{i} \sqrt{\widehat{a}_{i}^{2} X^{2}} \leq B_{i} . \quad$ The resulting Ellipsoid-RO model is as follows:

(Ellipsoid-RO model)

$\min Z_{e}$

$$
\begin{aligned}
& C_{f}+C_{D}\left(D_{j}^{0}\right)+\Omega P \leq Z_{e} \\
& P \geq \sqrt{\sum_{j=1}^{n} \widehat{D}_{j}^{2} r_{i j}^{2}, \quad \forall i \in I, \forall j \in J} \\
& r_{i} \geq \sum_{i=1}^{m} \sum_{j=1}^{n} y_{i j} c_{e}
\end{aligned}
$$$$
\text { s.t. } \quad \sum_{i=1}^{m} y_{i j}=1, \quad \forall j \in J
$$

$$
\begin{aligned}
& \sum_{j=1}^{n} D_{j}^{0} y_{i j}+\Omega P \leq H_{i}^{w}, \quad \forall i \in I \\
& \left\lceil y_{i j}\right\rceil \cdot\left(\frac{d_{i j}}{\bar{v}_{j}}\right) \leq T_{t}^{\mathrm{Max}}, \quad \forall i \in I, \forall j \in J \\
& C_{v}\left(x_{i}, D_{j}\right)+\Omega^{\prime} P \leq E_{e}^{\mathrm{Max}}, \quad \forall i \in I, \forall j \in J \\
& 0 \leq y_{i j} \leq 1, \quad \forall i \in I, \forall j \in J \\
& 0 \leq x_{i} \leq 1, \quad \forall i \in I .
\end{aligned}
$$

Proof. Constraint (19) is equivalent to constraint (14).

$$
a_{i}^{T} X+\Omega_{i} \sqrt{\widehat{a}_{i}^{2} X^{2}} \leq B_{i}
$$

The uncertainty ellipsoid uncertainty set is defined as follows: 


$$
U^{E}=\left\{a_{i} \in R^{n}:\left(a_{i}-\bar{a}_{i}\right)^{T} \Sigma^{-1}\left(a_{i}-\bar{a}_{i}\right) \leq \Omega^{2}\right\} .
$$

Among them $\bar{a}_{i}$ is nominal value, $\Sigma$ is positive definite matrix, $\Omega$ is SP. It can be converted to a sphere with radius of $\Omega$.

$$
U^{E}=\left\{a_{i} \in R^{n}: a_{i}=\bar{a}_{i}+\Delta \xi, \xi \leq \Omega\right\} .
$$

And, where $\Delta=\sum^{1 / 2}$, the constraints $\max a_{i}^{T} X \leq B$ of (6) can be translated.

$$
\max \left\{a_{i}^{T} X:\left(a_{i}-\bar{a}_{i}\right)^{T} \Sigma^{-1}\left(a_{i}-\bar{a}_{i}\right) \in \Omega^{2}\right\} .
$$

As for $\Sigma$ being positive, $\max \left\{a_{i}^{T} X:\left(a_{i}-\bar{a}_{i}\right)^{T} \Sigma^{-1}\left(a_{i}-\right.\right.$ $\left.\left.\bar{a}_{i}\right) \in \Omega^{2}\right\}$ is a convex problem. Therefore, LP can be solved by Karush-Kuhn-Tucher (KKT) condition. Set $a_{i}^{*}$ is an optimal solution, $u^{*}$ is Lagrange multiplier, and $z^{*}$ is an optimal value, then,

$$
\begin{aligned}
\min \mathscr{F}\left(a_{i}^{*}\right) & =-a_{i}^{* T} X \\
\text { s.t. } g\left(a_{i}^{*}\right) & =\left(a_{i}^{*}-\bar{a}_{i}\right)^{T} \Sigma^{-1}\left(a_{i}^{*}-\bar{a}_{i}\right)-\Omega^{2} \leq 0 .
\end{aligned}
$$

According to KKT condition,

$$
\begin{aligned}
& L\left(a_{i}^{*}, u_{i}^{*}\right)=f\left(a_{i}^{*}\right)+u_{i}^{*} g\left(a_{i}^{*}\right), \\
& \text { s.t. } \frac{d L\left(a_{i}^{*}, u_{i}^{*}\right)}{d a_{i}^{*}}=0 ; \quad a\left(a_{i}^{*}\right)=0 ; u^{*} \geq 0, \\
& L\left(a_{i}^{*}, u_{i}^{*}\right)=-a_{i}^{* T} X+u^{*}\left[\left(a_{i}^{*}-\bar{a}_{i}\right)^{T} \Sigma^{-1}\left(a_{i}^{*}-\bar{a}_{i}\right)-\Omega^{2}\right], \\
& \text { s.t. }-X+2 u^{*} \Sigma^{-1}\left(a_{i}^{*}-\bar{a}_{i}\right)=0, \\
& u^{*}\left[\left(a_{i}^{*}-\bar{a}_{i}\right)^{T} \Sigma^{-1}\left(a_{i}^{*}-\bar{a}_{i}\right)-\Omega^{2}\right]=0, \\
& u^{*} \geq 0 .
\end{aligned}
$$

So,

$$
\begin{array}{r}
a_{i}^{*}-\bar{a}_{i}=\frac{\Sigma X}{2 u^{*},} \\
{\left[\left(\frac{\Sigma X}{2 u^{*}}\right)^{T} \Sigma^{-1}\left(\frac{\Sigma X}{2 u^{*}}\right)\right]=\Omega^{2} .}
\end{array}
$$

Then,

$$
\begin{aligned}
{\left[\left(\frac{\Sigma X}{2 u^{*}}\right)^{T} \Sigma^{-1}\left(\frac{\Sigma X}{2 u^{*}}\right)\right] } & =\Omega^{2} \\
& \Rightarrow \frac{1}{2 u^{*}} X^{T} \Sigma^{T} \Sigma^{-1} \frac{1}{2 u^{*}} \Sigma X=\Omega^{2} \\
& \Rightarrow \frac{1}{\left(2 u^{*}\right)^{2}} X^{T} \Sigma X=\Omega^{2} \\
& \Rightarrow \frac{1}{\left(2 u^{*}\right)^{2}}=\frac{\Omega^{2}}{X^{T} \Sigma X} \\
& \Rightarrow \frac{1}{2 u^{*}}=\frac{\Omega}{\sqrt{(X / 2)^{T} \Sigma(X / 2)}} .
\end{aligned}
$$

From (25) and (26), we get the following:

$$
a_{i}^{*}=\bar{a}_{i}+\frac{\Omega}{2 \sqrt{(X / 2)^{T} \Sigma(X / 2)}} \Sigma X=\bar{a}_{i}+\frac{\Omega}{\sqrt{X^{T} \Sigma X}} \Sigma X .
$$

Therefore,

$$
\begin{aligned}
& z^{*}=X^{T} a_{i}^{*}+X^{T} \frac{\Omega}{\sqrt{x^{T} \Sigma x}} \Sigma X, \\
& z^{*}=X^{T} a_{i}^{*}+\Omega \sqrt{X^{T} \Sigma X} .
\end{aligned}
$$

Since $\Sigma_{i}$ is a diagonal matrix of elements $\widehat{a}_{i}^{2}$, then,

$$
\begin{aligned}
& a_{i}^{T} X+\Omega_{i} \sqrt{X^{T} \Sigma_{i} X} \leq B_{i} \\
& \Rightarrow a_{i}^{T} X+\Omega_{i} \sqrt{\hat{a}_{i}^{2} X^{2}} \leq B_{i} .
\end{aligned}
$$

Above all, Theorem 3 can be proved.

\section{Case Analysis}

In this section, an example is given to verify the effectiveness of the robust optimization method in solving the routing planning problem of HHC. In this paper, the HHC enterprise in Tangshan (China) is selected as a sample (Figures 2 and 3). The company is engaged in HHC and nursing assistance services to provide services for some communities in Tangshan city. The company is faced with double-level planning problems in the operation process.

The first level of planning is to determine the location of the nursing station. On the basis of comprehensive consideration of various location factors, the HHC and nursing service company selected the following 6 nodes as alternative nursing stations, namely, Concord nursing station, Fengnan nursing station, workers' nursing station, affinity source nursing station, Fule nursing station, and Kaiping nursing station, which are represented by $N_{1}, N_{2}, \ldots, N_{6}$ in the following article. So, the first level of planning is to determine the choice of these nursing stations.

The second level is routing planning. In this medical nursing system, there are 6 alternative nursing stations, 8 demand communities, and 48 alternative routings corresponding to different operating costs. The eight demand communities are Luanxing community, Hongda community, Fulianyuan, Shengtai manor, Tianheyuan community, Jiarun Rainbow City, Guzhen new town, and Yuehua new building, which are expressed in $D_{1}, D_{2}, \ldots, D_{8}$ later. The setting of transportation cost is based on the comprehensive calculation of the real-time oil price in Tangshan, the actual distance between the demand community and the nursing station. It even involves factors such as traffic congestion and time limit. Therefore, the choice of routing is second level planning based on the comprehensive consideration of relevant costs.

4.1. Related Data. The basic data information includes the nominal demand of the demand community, the maximum service supply of the medical care and nursing station, the 


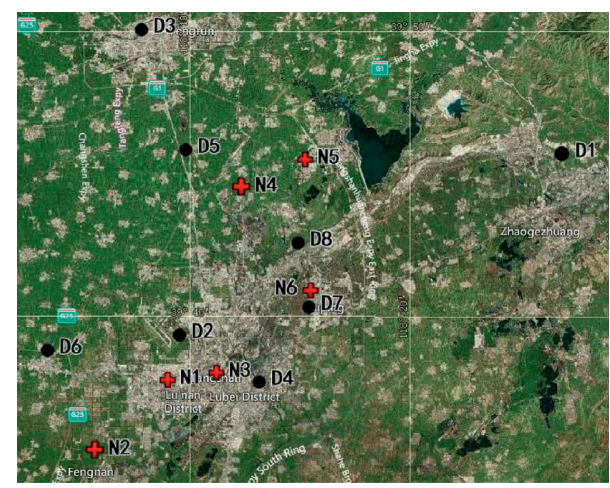

Figure 2: Actual map location.

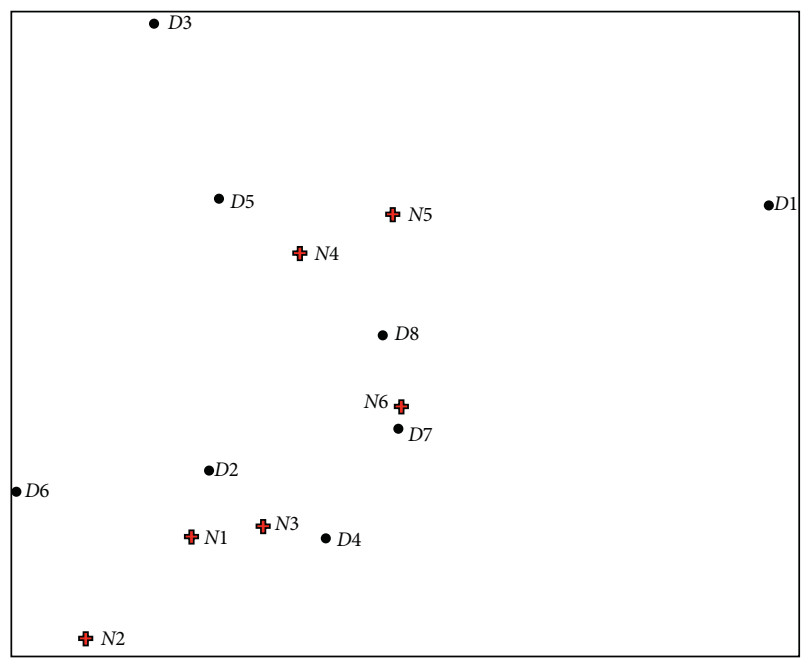

FIgURE 3: Relative location.

traffic conditions of the area, and the fixed operating costs, as shown in Table 2.

The actual distance between nodes obtained directly through Google map is shown in Table 3. Other parameters of the model are as follows: the benchmark door-to-door service time is 20 mins, the time delay cost is $10 \mathrm{CNY} /$ hour, and the maximum delay time is 60 mins. If it is overdue, the demand community will cancel the demand service order. The carbon tax is $2.1 \mathrm{CNY} / \mathrm{kg}$, and the fuel consumption per unit distance vehicle is $8.33 \mathrm{~L} / 100 \mathrm{~km}$. In addition, the $\mathrm{CO}_{2}$ emission coefficient is $0.16 \mathrm{~L} / \mathrm{h}{ }^{*} t$, and the $\mathrm{CO}_{2}$ emission factor is $2.51 \mathrm{~kg} / \mathrm{L}$ [42].

4.2. Optimal Solution. In this section, we use MATLAB as the programming platform, use Gurobi (9.0.2) programming to solve the above model and compare LO with three RO models.

4.2.1. Algorithm and Optimal Result of Models. The specific calculation process is shown in Table 4:

Through the above algorithm, the optimal solution of the LO model is $7.2665 E+03 \mathrm{CNY}$, time consuming $4.535591 \mathrm{~s}$. The routing planning scheme of the LO model is shown in
TABLE 2: Basic parameters.

\begin{tabular}{lcccccccc}
\hline Communities & $D_{1}$ & $D_{2}$ & $D_{3}$ & $D_{4}$ & $D_{5}$ & $D_{6}$ & $D_{7}$ & $D_{8}$ \\
\hline Nominal demand & 125 & 95 & 140 & 135 & 160 & 155 & 175 & 130 \\
HHC station & $N_{1}$ & $N_{2}$ & $N_{3}$ & $N_{4}$ & $N_{5}$ & $N_{6}$ & - & - \\
Maximum service & 225 & 260 & 240 & 235 & 180 & 225 & - & - \\
Average speed & 45 & 40 & 50 & 65 & 35 & 40 & - & - \\
Fixed costs & 750 & 650 & 550 & 450 & 420 & 480 & - & - \\
\hline
\end{tabular}

TABLE 3: Distance between nodes.

\begin{tabular}{ccccccccc}
\hline$d_{i j}$ & $D_{1}$ & $D_{2}$ & $D_{3}$ & $D_{4}$ & $D_{5}$ & $D_{6}$ & $D_{7}$ & $D_{8}$ \\
\hline$N_{1}$ & 35.40 & 5.40 & 2.40 & 8.10 & 17.90 & 11.60 & 14.70 & 16.90 \\
$N_{2}$ & 36.80 & 13.30 & 8.50 & 15.50 & 29.10 & 10.80 & 23.90 & 24.90 \\
$N_{3}$ & 33.10 & 5.90 & 6.50 & 4.50 & 17.70 & 14.00 & 10.80 & 18.20 \\
$N_{4}$ & 26.90 & 14.60 & 22.10 & 15.10 & 6.10 & 22.00 & 12.30 & 7.30 \\
$N_{5}$ & 19.80 & 23.80 & 29.10 & 22.10 & 12.50 & 34.80 & 15.10 & 9.70 \\
$N_{6}$ & 24.10 & 12.20 & 15.70 & 10.40 & 17.20 & 26.00 & 4.20 & 5.50 \\
\hline
\end{tabular}

TABle 4: Algorithm steps of LO model.

$\begin{array}{cc}\text { Step } 1 & \begin{array}{c}\text { Input initial value and relevant parameters; } \\ \text { Input parameter variable constraints, carbon emission } \\ \text { constraints, time window constraints, and other } \\ \text { Constraints; }\end{array} \\ \text { Step } 2 & \begin{array}{r}\text { Set the solution environment and solve it through } \\ \text { the solver Gurobi; }\end{array} \\ \text { Step } 4 & \begin{array}{r}\text { If step } 2 \text { is satisfied, terminate, no, execute step 1; } \\ \text { Step 5 }\end{array} \\ \text { Output the optimal solution and running time. }\end{array}$

Figure 4. The wider the routing line width is, the larger the proportion of this routing is. As can be seen from Figure 4, in the second level of routing planning, $\mathrm{HHC}$ and nursing stations are the main ones, accounting for $38.3 \%$ and $32.5 \%$ of the total demand, respectively, and they are responsible for the supply of main service personnel. Although this can ensure the stable supply, there is a certain space for improvement in the routing planning, such as over long-distance service, which increases the transportation cost in a large amount that is not conducive to the operation of the company, so it is necessary to optimize the distribution routing.

The algorithm is shown in Table 5. And the results of the models are shown in Table 6.

4.2.2. Box-RO Model. In the Box-RO model, the influence of $\Psi$ on the lowest total cost is constantly changing. The calculation results of the Box-RO model are shown in Table 6. The total cost of the HHC care planning network raises with the trend of increase. When $\Psi=0$, the model is equivalent to the LO model, and the total cost is $7.6213 E+03$ CNY. As shown in Figure 5, the Box-RO model is similar to the LO model: (i) In the selection of nursing sites, the first level planning needs to add two nodes, from 4 nursing stations to 6 nursing stations. (ii) In the second layer of routing planning, the number of routings increases from 16 to 19 . The routing planning is more complex, and its total cost also increases. As a whole, there are many long-distance transmission lines. 


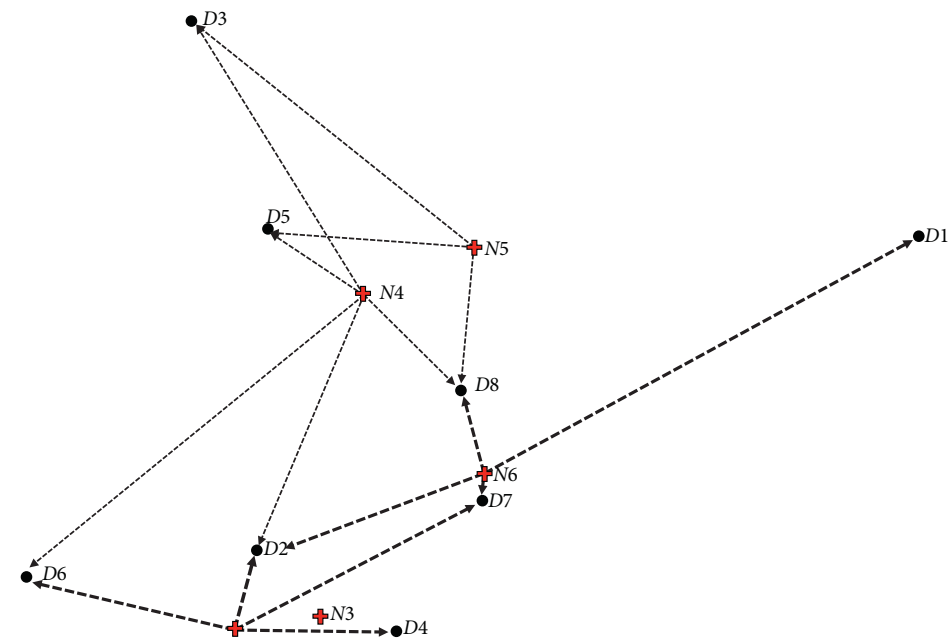

$\stackrel{2 \mathrm{~km}}{\text { Opencyclemap }}$

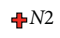

FIgUre 4: Distribution routing in LO model.

TABle 5: Algorithm steps of RO model.

Step 1

Input initial value, given the parameters:

Step 2

$U^{B}=\left\{\varepsilon:\|\varepsilon\|_{\infty} \leq \Psi\right\}=\left\{\varepsilon:\left|\varepsilon_{j}\right| \leq \Psi\right\} ; U^{P}=\left\{\varsigma:\|\varepsilon\|_{1} \leq \Gamma\right\}=\left\{\varepsilon: \sum\left|\varepsilon_{j}\right| \leq \Gamma_{j}\right\} ; U^{E}=\left\{\varsigma:\|\varepsilon\|_{2} \leq \Omega\right\}=\left\{\varepsilon \sqrt{\sum_{J} \varepsilon_{j}^{2}} \leq \Omega\right\} ;$

Step 3

If the random number satisfies the condition, step 3; otherwise, return step 1;

Input parameter and constraints;

Step 4

\#1 Box-RO and Polyhedron-RO model direct input constraints;

Step 5

\#2 Ellipsoid-RO after the model further relax the parameter constraints;

If meets step 3, terminate; else, execute step 1;

Step 6

Set the solution environment and solve it through the solver Gurobi; Output the optimal solution and running time.

TABLe 6: Operation results of RO models.

\begin{tabular}{|c|c|c|c|c|c|c|}
\hline \multirow[b]{2}{*}{ SP } & \multicolumn{2}{|c|}{ Box-RO model } & \multicolumn{2}{|c|}{ Polyhedron-RO model } & \multicolumn{2}{|c|}{ Ellipsoid-RO model } \\
\hline & Total cost & Time & Cost & Time & Cost & Time \\
\hline 0 & $7.2665 E+03$ & 4.747611 & $7.2665 E+03$ & 4.688161 & $7.2665 E+03$ & 4.493691 \\
\hline 1 & $7.8087 E+03$ & 4.297063 & $7.6183 E+03$ & 4.454975 & $7.4332 E+03$ & 4.348147 \\
\hline 2 & $7.8420 E+03$ & 4.471258 & $7.6719 E+03$ & 4.528155 & $7.4520 E+03$ & 5.021394 \\
\hline 3 & $7.9285 E+03$ & 4.146447 & $7.8808 E+03$ & 4.411533 & $7.5019 E+03$ & 4.091843 \\
\hline 4 & $7.9541 E+03$ & 4.098501 & $7.9590 E+03$ & 4.387638 & $7.5282 E+03$ & 4.187512 \\
\hline 5 & $8.0119 E+03$ & 4.602096 & $8.1480 E+03$ & 4.467358 & $7.6244 E+03$ & 4.949629 \\
\hline 6 & $8.1215 E+03$ & 4.465805 & $8.4583 E+03$ & 4.248660 & $7.7855 E+03$ & 4.498152 \\
\hline 7 & $8.1620 E+03$ & 4.049612 & $8.5868 E+03$ & 4.267902 & $7.9260 E+03$ & 4.522854 \\
\hline 8 & $8.2755 E+03$ & 4.012588 & $9.0733 E+03$ & 4.258624 & $8.1003 E+03$ & 4.320861 \\
\hline
\end{tabular}

4.2.3. Polyhedron-RO Model. In the Polyhedron-RO model, the impact of $\Gamma$ on the total cost is constantly changing. The calculation results of the Polyhedron-RO model are shown in Table 6. The total cost of the HHC care planning network raises with the trend of increase $\Gamma$. When $\Gamma=0$, Polyhedron-RO model is equivalent to the LO model. As shown in Figure 6, the Polyhedron-RO model compares the Box-RO model with the LO model: (i) In the first level planning, there are two more nursing stations than the LO model. (ii) In the second layer of planning, the number of routings is increased to 21 compared with the Box-RO model. In terms of the proportion of each routing, the proportion of long-distance line transportation is reduced and the proportion of short-distance transportation is increased, which has a positive role in promoting the routing optimization. 


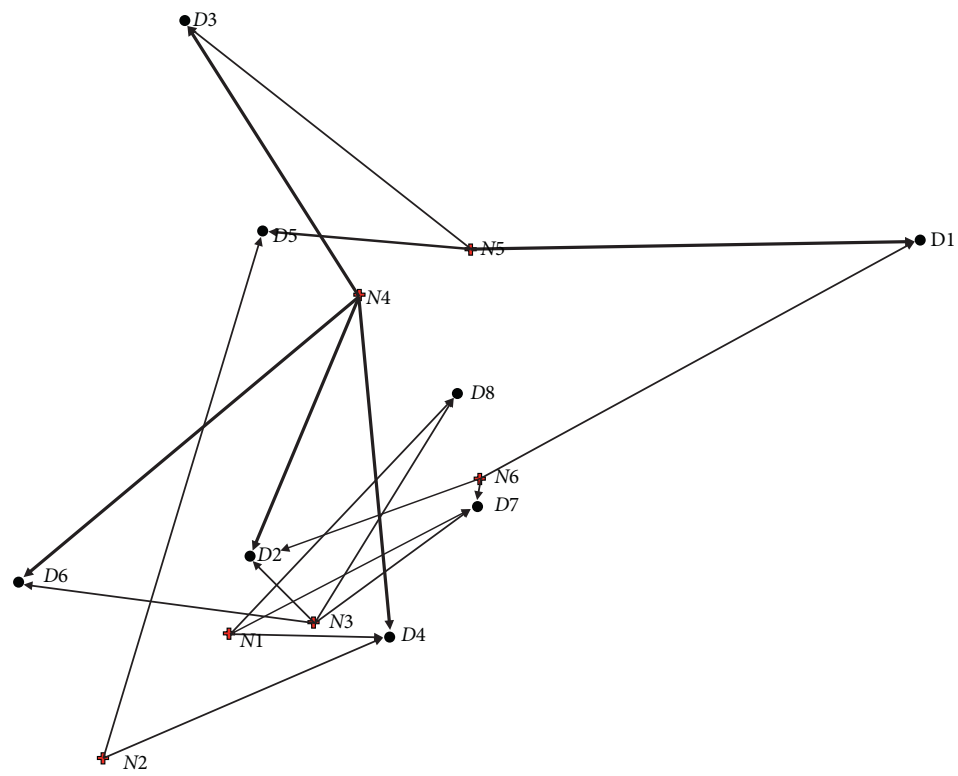

FIgURE 5: Routing planning of Box-RO model.

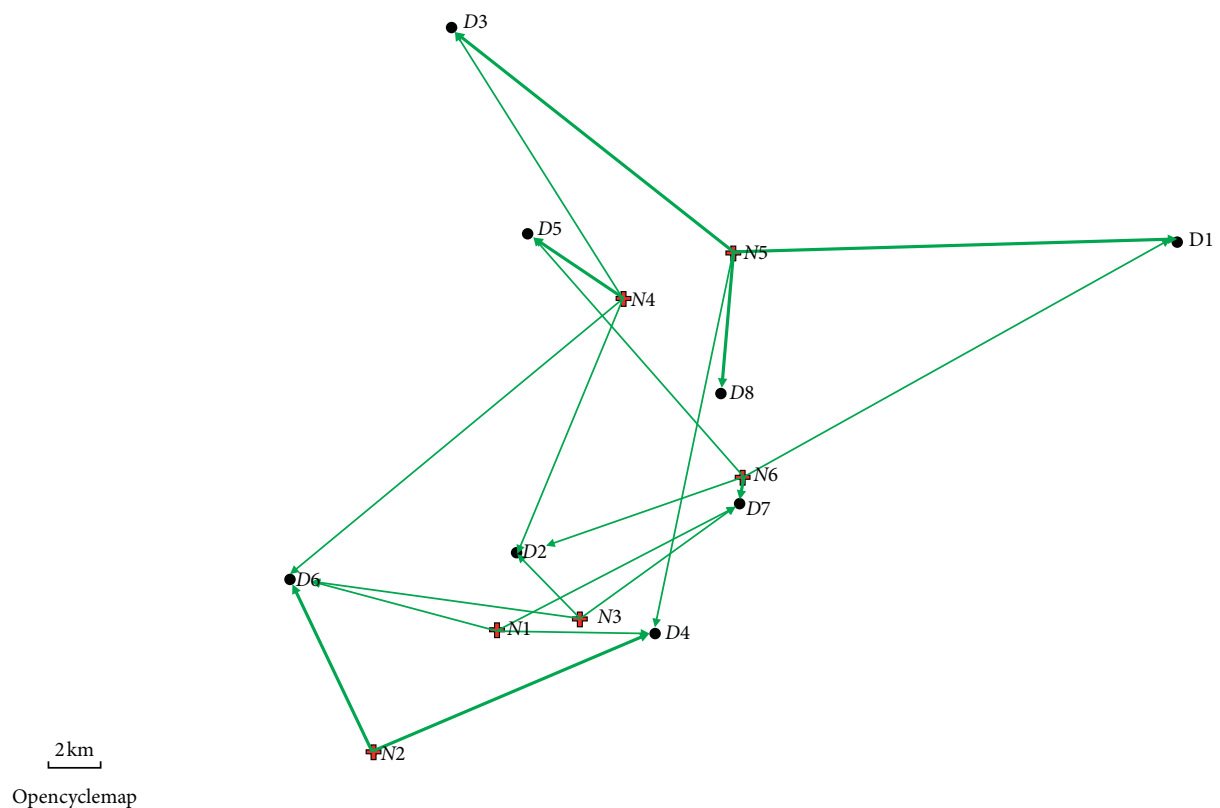

FIgure 6: Routing of Polyhedron-RO model.

4.2.4. Ellipsoid-RO Model. In the Ellipsoid-RO model, the effect of the security parameter $\Omega$ on the lowest total cost is changing. The calculation results of the Ellipsoid-RO model are shown in Table 6. Similarly, the total cost of the HHC care planning network increases with the increase of $\Omega$. At that time, $\Omega=0$, the Ellipsoid-RO model was equivalent to the LO model, with the same total cost. The difference between the Ellipsoid-RO model, Box, and Polyhedron-RO model is as follows.

The Ellipsoid-RO model's route planning plan is shown in Figure 7. In the first stage planningBox $=6 \Leftrightarrow$ Polyhedron
$=6>$ Ellipsoid $=5>\operatorname{MILP}=4$, and 5 nursing stations are selected for the Ellipsoid-RO model. By comparison, it is found that in the RO model, the performance is the best, next to the LO model. In the second level planning, the number of routings is reduced to 18. The proportion of service in each routing shows a trend of transferring to short-distance routing, and the proportion of HHC service supply undertaken by short-distance routing increases. Therefore, the first stage of vehicle mileage efficiency is higher; the second stage of the distribution route is more accurate, showing better optimization performance. 


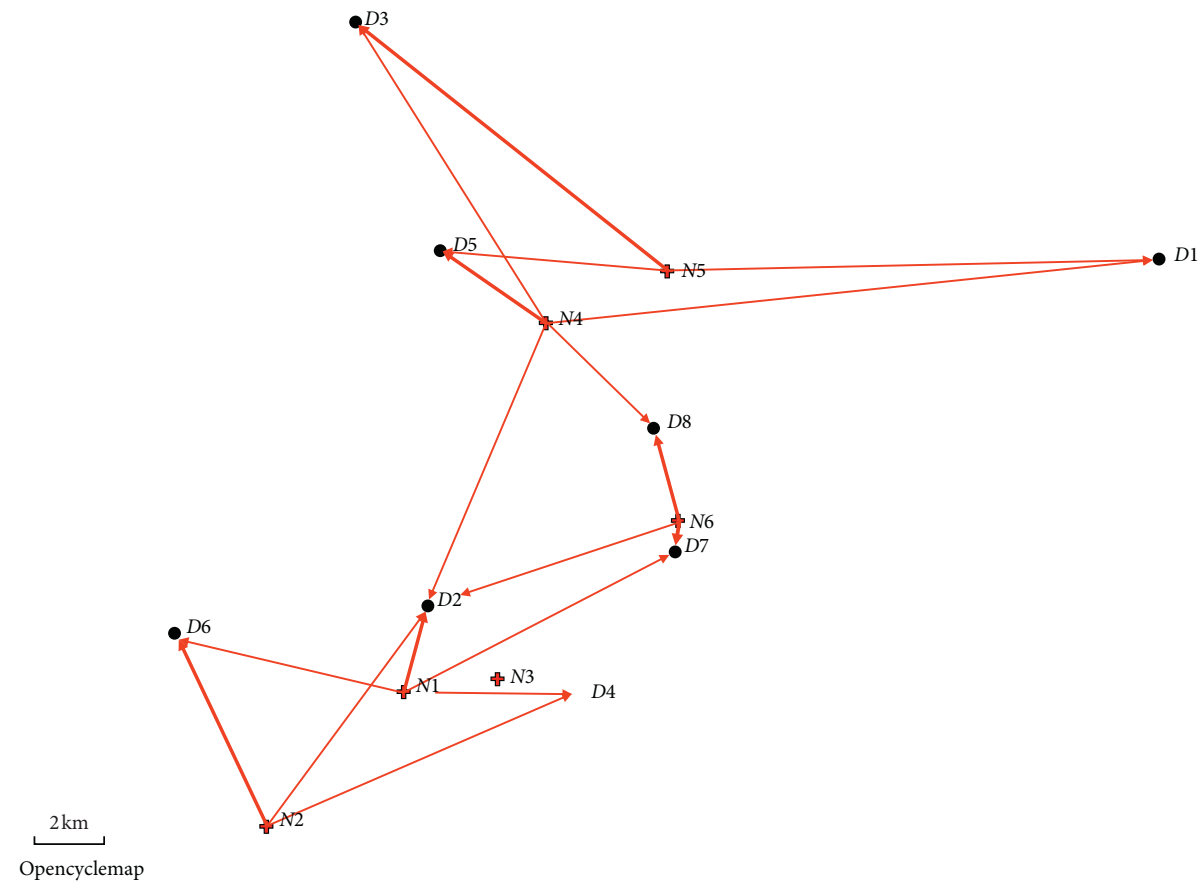

FIGURE 7: Routing scheme of Ellipsoid-RO model.

4.3. Sensitivity Analysis. This section compares the performance of each model, including efficiency, uncertainty, and the degree of demand fluctuation.

4.3.1. Efficiency Comparison. This section analyzes the operation efficiency of the four models. In order to take comparison conveniently, run the model in the same computer environment at the same time, we set the safety parameter as the only variable, and then observe the running time of the model.

Figure 8 shows the operational efficiency of the four robust optimization models. Among them, the Box-RO model has the highest operation efficiency and the fastest convergence speed (lower than the LO model as a whole: $4.535591 \mathrm{~s})$. The Polyhedron-RO model is the most stable with little fluctuation (the peak value is $4.688161 \mathrm{~s}$, the low value is $4.24866 \mathrm{~s}$ ). The operation fluctuation degree of the robust optimization model of Ellipsoid is the largest (the peak is five-point zero two one three nine four $s$. The low value is $4.091843 \mathrm{~s}$ ) maximum amplitude is $0.9296 \mathrm{~s}$. Due to the small scale, there is little difference in the calculation time. However, when the constraints and variables in the model increase to tens of thousands or even tens of thousands, the operation efficiency will be significantly different.

4.3.2. Impact of Carbon Emission Restrictions. Through the above comparative analysis, it is found that the performance of the Ellipsoid-RO model is superior. Therefore, under the Ellipsoid-RO model, the impact of carbon emissions on the total cost is analyzed. Under the same parameters $(\varepsilon=0.10, \Omega=4)$, with the change of carbon tax as the only variable, the calculation results are shown in Table 7. When

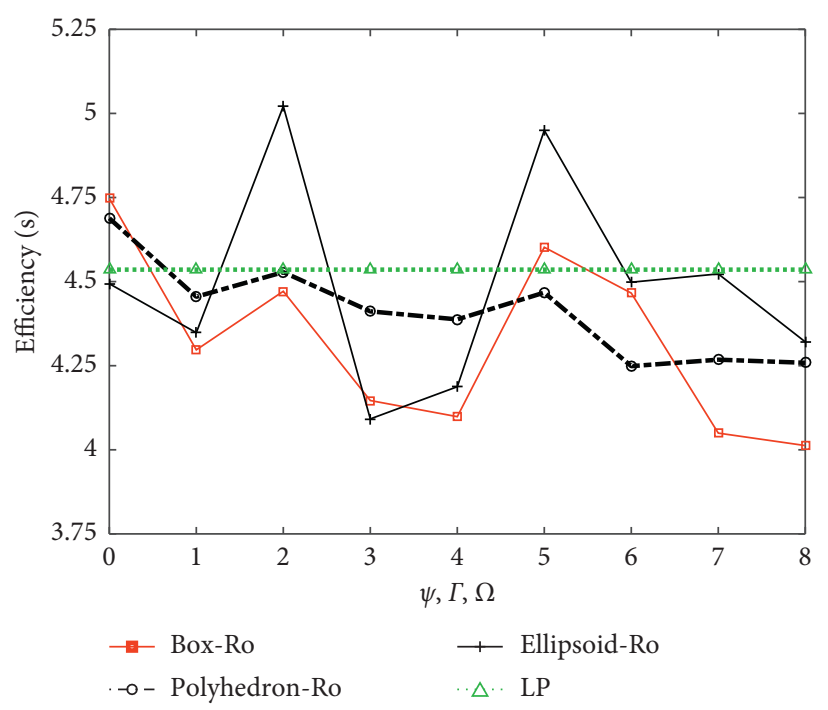

FIgURE 8: Comparison of model efficiency.

the carbon tax increases by $25 \%(2.0-2.50)$, the cost of using robust optimization model decreases by $8.274 \%$.

With the increase of carbon emission tax, the HHC enterprises have to change their own routing distribution scheme and change the focus of resource allocation from $N_{2}, N_{5}$ to $N_{4}, N_{6}$. The main reason is that the carbon emission cost of $N_{4}, N_{6}$ vehicles driving in the relatively close distance to the demand community can be significantly reduced. Fortunately, the total cost of distribution also shows a downward trend when we choose a closer route. In this way, we can not only save the cost of product distribution and transportation but also get better environmental benefits, which is conducive to the sustainable development of the ecological environment. 
TABLE 7: The impact of carbon emission.

\begin{tabular}{lcccccc}
\hline $\begin{array}{l}\text { Carbon } \\
\operatorname{tax}\end{array}$ & Cost & $N_{1}$ & $N_{2}$ & $N_{4}$ & $N_{5}$ & $N_{6}$ \\
\hline 2.00 & $7.5282 E+03$ & $25.89 \%$ & $22.03 \%$ & $18.35 \%$ & $15.34 \%$ & $18.39 \%$ \\
2.05 & $7.6461 E+03$ & $27.13 \%$ & $21.78 \%$ & $18.45 \%$ & $14.09 \%$ & $18.55 \%$ \\
2.10 & $7.6522 E+03$ & $28.56 \%$ & $21.45 \%$ & $18.69 \%$ & $13.18 \%$ & $18.12 \%$ \\
2.15 & $7.6816 E+03$ & $29.75 \%$ & $21.16 \%$ & $19.04 \%$ & $12.45 \%$ & $17.60 \%$ \\
2.20 & $7.4781 E+03$ & $30.08 \%$ & $20.57 \%$ & $19.08 \%$ & $11.12 \%$ & $19.15 \%$ \\
2.25 & $7.4197 E+03$ & $30.79 \%$ & $19.34 \%$ & $20.13 \%$ & $10.13 \%$ & $19.61 \%$ \\
2.30 & $7.2870 E+03$ & $31.09 \%$ & $18.94 \%$ & $21.76 \%$ & $9.45 \%$ & $18.76 \%$ \\
2.35 & $7.2014 E+03$ & $31.25 \%$ & $18.21 \%$ & $22.48 \%$ & $8.07 \%$ & $19.99 \%$ \\
2.40 & $7.1650 E+03$ & $31.76 \%$ & $17.09 \%$ & $23.93 \%$ & $7.96 \%$ & $19.26 \%$ \\
2.45 & $6.9652 E+03$ & $32.26 \%$ & $16.72 \%$ & $24.17 \%$ & $7.13 \%$ & $19.72 \%$ \\
2.50 & $6.9053 E+03$ & $32.97 \%$ & $16.18 \%$ & $24.46 \%$ & $6.23 \%$ & $20.16 \%$
\end{tabular}

4.3.3. Impact of Demand Fluctuation and SP. In this section, the impact of demand fluctuation on total cost in three RO models is compared and analyzed. Under the condition of fixed safety parameter $(\psi=\Gamma=\Omega=4)$, the impact of fluctuation on cost is explored. The calculation results are shown in Figure 9. Although the total cost of the RO model is higher than that of the deterministic LO model, even in the worst situation, the routing planning scheme can still be given. In addition, the increasing trend and proportion are quite different. Among them, the Polyhedron-RO model and Box-RO model have greater randomness, and the EllipsoidRO model has strong ability to resist uncertainty. Careful observation shows that the growth rate is slightly different. The cost of the Polyhedron-RO model increases sharply, while that of the Ellipsoid-RO model increases slowly.

Figure 10 analyzes the impact of the change of safety parameters on the total cost, and it can be seen that with the increase in SP, the overall logistics distribution cost shows an upward trend. Different RO models have different rising rates of cost, and the LO model is not affected by the safety parameters and maintains a low level; Polyhedron-RO model has the highest increasing rate of cost, and the most robust cost is paid for improving the safety level; Box-RO model is in the middle, and Ellipsoid model is the most stable, and lowest robust cost is paid for increasing SP.

\subsubsection{Service Level and Responsiveness of Routing Planning.} In this section, three robust optimization models are analyzed by the level of service (SL). Due to the high requirements for the arrival time of service personnel in the process of $\mathrm{HHC}$ care, this section compares the service quality level of the model through the time difference and analyzes the advantages and disadvantages of different models. The calculation of service level is as follows:

$$
S=\frac{\sum_{I, J}\left(1-\left(y_{i j} \widetilde{D}_{j} d_{i j} / \bar{v}_{i}-t\right) / t\right)}{(I+J) \sum_{J} \widetilde{D}_{j}} \times 100 \%,
$$

where $I, J$ represents the number of arcs in the model. The computer simulation results under different parameters are shown in Figures 11 and 12.

Figure 11 shows the influence of random demand fluctuation on the model under the condition of fixed safety
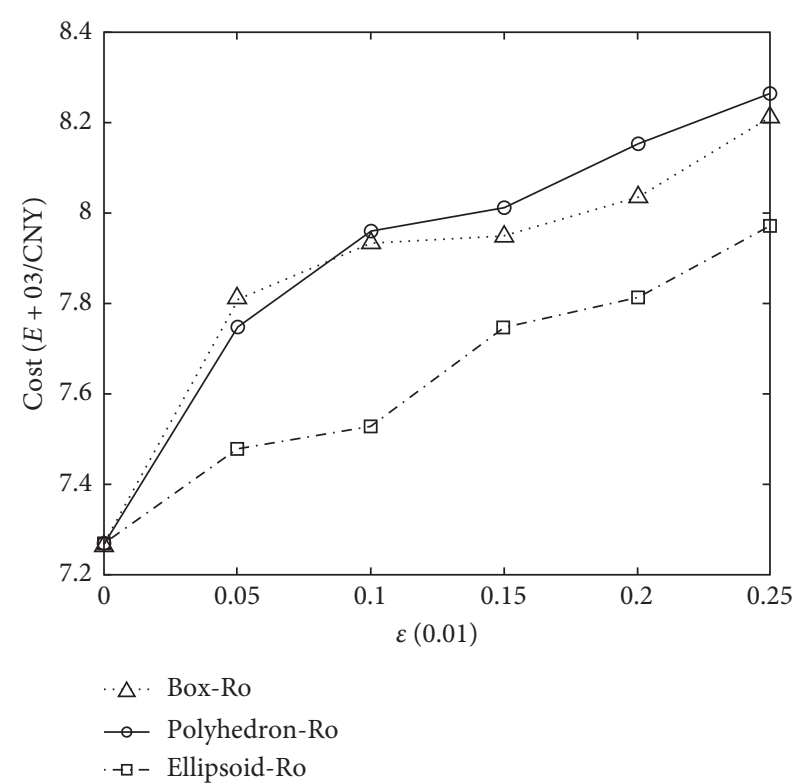

FIGURE 9: The influence of fluctuation.

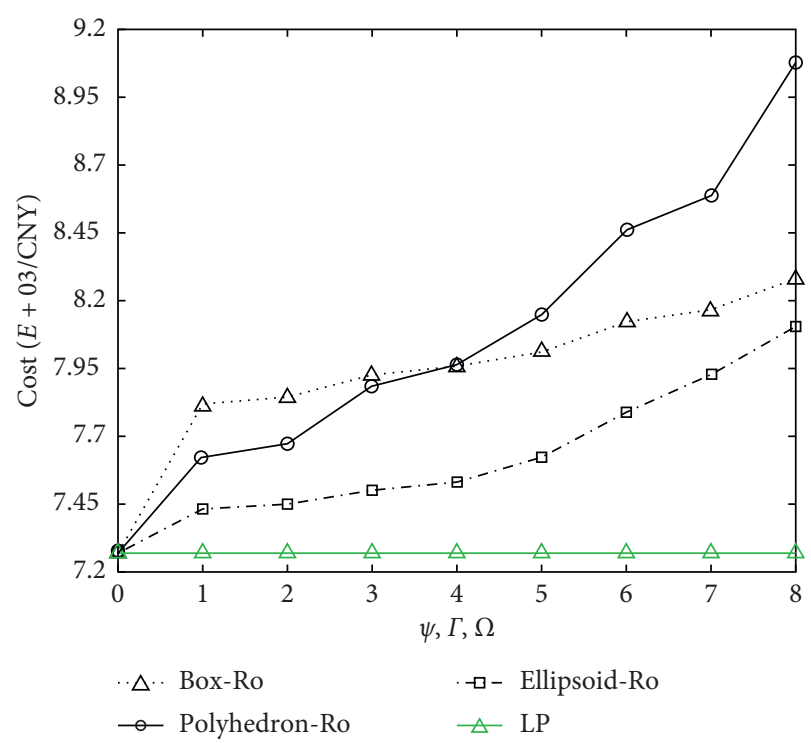

FIGURE 10: Influence of SP.

parameters $(\Psi=\Gamma=\Omega=3$ ), and the following conclusions can be obtained: (i) The service level of the LO model is not affected by random parameters, and of course, it cannot solve the routing planning problem under uncertainty. However, as the data are all determined, the service level is also the highest ( $\mathrm{SL}=93.38 \%$ ). (ii) The service level of the three RO models shows a downward trend with the increase of random demand volatility. The larger the amplitude of random parameter fluctuation, the lower the service level. (iii) Different RO models are also affected by uncertain parameters. The relative ratio shows that the Ellipsoid-RO model has strong robustness, the Polyhedron-RO model is in the middle, the Box-RO model is the most affected by volatility, which means that Box-RO model has the weakest 


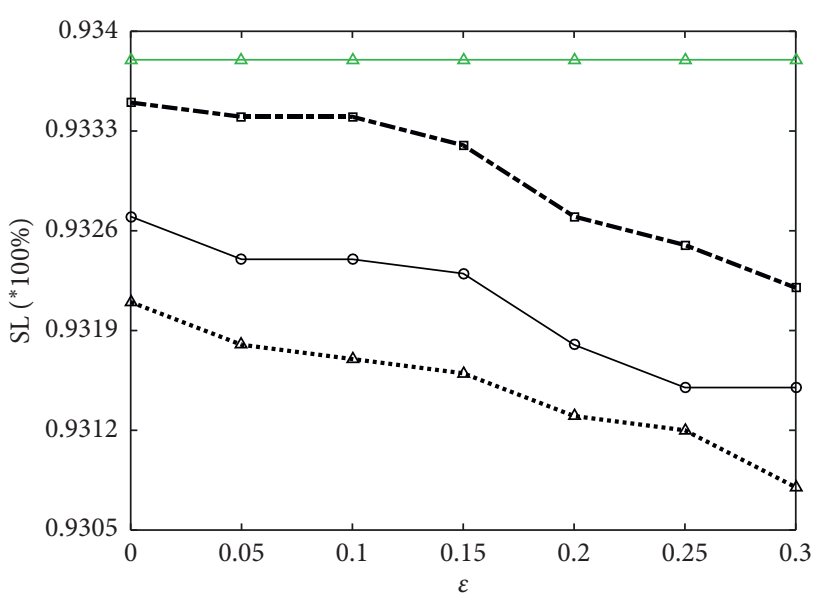

$\begin{array}{ll}\cdot \triangle \cdot \text { Box-Ro } & \cdot \square-\text { Ellipsoid-Ro } \\ \neg \text { - Polyhedron-Ro } & \triangle \text { LP }\end{array}$

FIGURE 11: SL affected by demand volatility.

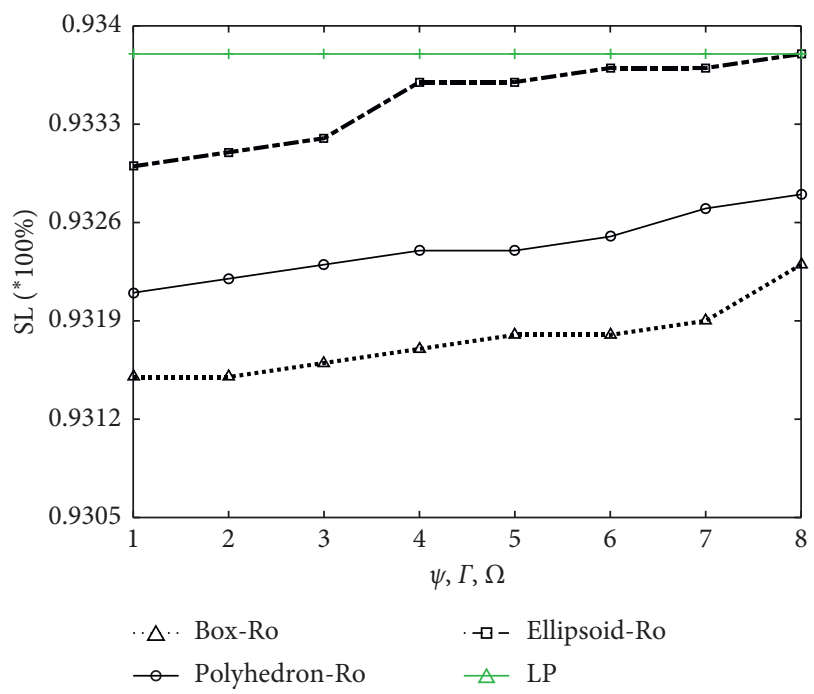

FIgURE 12: SL affected by safety parameters.

ability to resist changes in market environment, while the Ellipsoid-RO model has the best performance.

Figure 12 is an analysis of the impact of security parameters on the service level of the model under the condition of fixed random demand volatility $(\varepsilon=0.15)$. Fortunately, with the increase of security parameter level, service level shows an increasing trend. To a certain extent, it can make up for the robust cost (total cost increase) caused by uncertainty and also alleviate the loss of service level caused by stochastic demand volatility. Through careful comparison, it is found that the Ellipsoid-RO model still has strong robustness. When the security parameters increase from 1 to 8 , the logistics service level increases from $93.30 \%$ to $93.38 \%$, which is the same as the LO model. Therefore, in the actual market application, the decision maker of routing planning can determine the corresponding safety parameter level according to the preset expected service level. These studies have important reference significance for decision-making.
The following main conclusions can be drawn: (1) The basic LO model is idealized due to the market environment. In comparison, it can give the lowest total cost and the highest service level, but because the ideal data set is extremely difficult to obtain, or even unavailable, so the practical feasibility of the LO model is not high. (2) On the whole, three RO models will pay a certain robust price due to the impact of random demand fluctuations, such as increased costs, reduced service levels, and increased computational complexity, but they can solve the problem of uncertainties. The problem of path planning has a certain degree of robustness. (3) In terms of details, different RO models are also affected by uncertain demand parameters. In comparison, the Ellipsoid-RO model has strong robustness, the Polyhedron-RO model is centered, and the Box-RO model is most affected by volatility. This means that the BoxRO model has the weakest ability to resist changes in the market environment, while the Ellipsoid-RO model performs best. (4) Considering the large environmental background of the sustainable development of the economic environment, the carbon tax is studied as a cost, and it is found that when the carbon emission tax amount is increased, the company is also forced to choose a better distribution path, which not only benefits the company to save the cost but also can obtain certain environmental benefits. This discovery can provide some inspiration and reference for the low-carbon transformation and development of HHC enterprises.

\section{Conclusion}

Nowadays, China's economic development level is constantly improving, and the application of science and technology is constantly being updated. Especially under the severe situation that the proportion of the elderly population is constantly rising at present, the demand of all residents for community HHC is increasing accordingly. In addition, under the background of internationalization and globalization of the coordinated and sustainable development of the economic environment, the low-carbon economic model has also become an emerging development keyword for the HHC service industry. Therefore, from the perspective of a low-carbon economy, this study comprehensively considers fixed costs, transportation costs, time delay penalty costs, and carbon emission costs to explore path optimization management.

First, this paper establishes a basic linear optimization model. Under relatively ideal data, the programming algorithm is programmed through the MATLAB platform and finally solved by Gurobi with the feasible path planning scheme given. In order to prevent the alienation effect of uncertain demand parameters on the results, this article further splits random demand into nominal demand and random demand, and constructs corresponding uncertain sets, transforming linear optimization model into three robust optimization models, respectively, and then apply them to the path optimization problem of home health care service enterprise in Tangshan city. Through the case analysis of this paper, some valuable research results are 
obtained, which can provide some enlightenment and reference for the low-carbon transformation and development of family health care enterprises.

Although this study considers various major factors, there are still some shortcomings, for example, the application of information technology, Internet of technology, and $5 \mathrm{G}$ technology in the path planning of home health care services. In the future, our research will deeply discuss the application model of science and technology. It is foreseeable that the informatization and intelligence of the HHC service industry is the development trend in the future.

\section{Data Availability}

The data used to support the findings of this study are included within the article.

\section{Conflicts of Interest}

The authors declare that they have no conflicts of interest.

\section{Acknowledgments}

This work was supported by the research result of the Key Research Project of Social Science Development in Hebei Province, "The Pension Project of Home and Community Development in Hebei City" (No. 201802120101); the Natural Science Foundation of Hebei Province (No. G2019203387); Hebei Provincial Department of Human Resources and Social Security research project, "Internet+" home care platform service pricing research (No.JRS-20187024); the Research Project of Social Science Development in Hebei Province, "Study on Risk Management and Control of Electronic Medical Records in Grade III a hospitals" (No. 20200302092); and the Research Project of Social Science Development in Hebei Province, "Study on Stroke Risk Prediction Model Based on Data Mining Technology" (No. 20200303129).

\section{References}

[1] J. Jones, A. Wilson, H. Parker et al., "Economic evaluation of hospital at home versus hospital care: cost minimisation analysis of data from randomised controlled trial," Bmj, vol. 319, no. 7224, pp. 1547-1550, 1999.

[2] A. Errarhout, S. Kharraja, and C. Corbier, "Two-stage stochastic assignment problem in the home health care," IFACPapersOnLine, vol. 49, no. 12, pp. 1152-1157, 2016.

[3] J. Decerle, O. Grunder, A. Hajjam El Hassani, and O. Barakat, "A hybrid memetic-ant colony optimization algorithm for the home health care problem with time window, synchronization and working time balancing," Swarm and Evolutionary Computation, vol. 46, pp. 171-183, 2019.

[4] C. Fikar and P. Hirsch, "Home health care routing and scheduling: a review," Computers \& Operations Research, vol. 77, pp. 86-95, 2017.

[5] Y. Shi, T. Boudouh, and O. Grunder, "A robust optimization for a home health care routing and scheduling problem with consideration of uncertain travel and service times," Transportation Research Part E: Logistics and Transportation Review, vol. 128, pp. 52-95, 2019.
[6] E. Cho, K. Lee, D. Min, S. J. Chang, J. Kim, and H. Kim, "Development and validation of the nursing home care-related quality of life scale," Journal of the American Medical Directors Association, vol. 20, no. 11, pp. 1412-1418, 2019.

[7] K. Christensen, G. Doblhammer, R. Rau, and J. W. Vaupel, "Ageing populations: the challenges ahead," The Lancet, vol. 374, no. 9696, pp. 1196-1208, 2009.

[8] J. Wang, Y. Yu, and J. Tang, "Compensation and profit distribution for cooperative green pickup and delivery problem," Transportation Research Part B: Methodological, vol. 113, pp. 54-69, 2018.

[9] Yu Yang, S. Wang, J. Wang, and M. Huang, "A branch-andprice algorithm for the heterogeneous fleet green vehicle routing problem with time windows," Transportation Research Part B: Methodological, vol. 122, pp. 511-527, 2019.

[10] A. Bettinelli, V. Cacchiani, T. G. Crainic, and D. Vigo, "A branch-and-cut-and-price algorithm for the multi-trip separate pickup and delivery problem with time windows at customers and facilities," European Journal of Operational Research, vol. 279, no. 3, pp. 824-839, 2019.

[11] R. Menghi, A. Papetti, and M. Germani, "Product Service Platform to improve care systems for elderly living at home," Health Policy and Technology, vol. 8, no. 4, pp. 393-401, 2019.

[12] N. Maglaveras, V. Koutkias, I. Chouvarda et al., "Home care delivery through the mobile telecommunications platform: the Citizen Health System (CHS) perspective," International Journal of Medical Informatics, vol. 68, no. 1-3, pp. 99-111, 2002.

[13] E. Marcon, S. Chaabane, Y. Sallez, T. Bonte, and D. Trentesaux, "A multi-agent system based on reactive decision rules for solving the caregiver routing problem in home health care," Simulation Modelling Practice and Theory, vol. 74, pp. 134-151, 2017.

[14] S. E. Moussavi, M. Mahdjoub, and O. Grunder, "A matheuristic approach to the integration of worker assignment and vehicle routing problems: application to home healthcare scheduling," Expert Systems with Applications, vol. 125, pp. 317-332, 2019.

[15] M. F. Shore, "Medical leadership and the future of health care," The Annals of Thoracic Surgery, vol. 67, no. 5, pp. 1219-1226, 1999.

[16] L. Di Matteo, "The determinants of the public-private mix in Canadian health care expenditures: 1975-1996," Health Policy, vol. 52, no. 2, pp. 87-112, 2000.

[17] R. Ali, M. Mahdi Nasiri, F. Werner, M.M. Musavi, and F. Jolai, "The vehicle routing and scheduling problem with crossdocking for perishable products under uncertainty: two robust bi-objective models," Applied Mathematical Modelling, vol. 70, pp. 605-625, 2019.

[18] W. Zhang, M. Zhang, W. Zhang, Q. Zhou, and X. Zhang, "What influences the effectiveness of green logistics policies? A grounded theory analysis," Science of the Total Environment, vol. 714, Article ID 136731, 2020.

[19] C. Wang, Q. Zhang, and W. Zhang, "Corporate social responsibility, green supply chain management and firm performance: the moderating role of big-data analytics capability," Research in Transportation Business \& Management, vol. 37, no. 4, Article ID 100557, 2020.

[20] H.-G. Beyer and B. Sendhoff, "Robust optimization-A comprehensive survey," Computer Methods in Applied Mechanics and Engineering, vol. 196, no. 33-34, pp. 3190-3218, 2007.

[21] J. M. Mulvey, R. J. Vanderbei, and S. A. Zenios, "Robust optimization of large-scale systems," Operations Research, vol. 43, no. 2, pp. 264-281, 1995. 
[22] C. Hu, J. Lu, X. Liu, and G. Zhang, "Robust vehicle routing problem with hard time windows under demand and travel time uncertainty," Computers \& Operations Research, vol. 94, pp. 139-153, 2018.

[23] A. Ben-Tal and A. Nemirovski, "Robust optimization ? methodology and applications," Mathematical Programming, vol. 92, no. 3, pp. 453-480, 2002.

[24] D. Bertsimas, D. B. Brown, and C. Caramanis, "Theory and applications of robust optimization," SIAM Review, vol. 53, no. 3, pp. 464-501, 2011.

[25] D. Bertsimas and M. Sim, "The price of robustness," Operations Research, vol. 52, no. 1, pp. 35-53, 2004.

[26] D. Bertsimas and M. Sim, "Robust discrete optimization and network flows," Mathematical Programming, vol. 98, pp. 49-71, 2004.

[27] R. Montemanni and L. M. Gambardella, "A branch and bound algorithm for the robust spanning tree problem with interval data," European Journal of Operational Research, vol. 161, no. 3, pp. 771-779, 2005.

[28] A. Sarac, R. Batta, and C. M. Rump, "A branch-and-price approach for operational aircraft maintenance routing," European Journal of Operational Research, vol. 175, no. 3, pp. 1850-1869, 2006.

[29] X. Zhang, L. Li, S. Wang, and F. Yang, "Valiant load-balanced robust routing algorithm for multi-granularity connection requests in traffic-grooming WDM mesh networks," Computer Communications, vol. 30, no. 18, pp. 3498-3507, 2007.

[30] J. Huang and Q. Wang, "Robust optimization of hub-andspoke airline network design based on multi-objective genetic algorithm," Journal of Transportation Systems Engineering and Information Technology, vol. 9, no. 3, pp. 86-92, 2009.

[31] S. Shahnejat-Bushehri, R. Tavakkoli-Moghaddam, S. Momen, A. Ghasemkhani, and H. Tavakkoli-Moghaddam, "Home health care routing and scheduling problem considering temporal dependencies and perishability with simultaneous pickup and delivery," IFAC-PapersOnLine, vol. 52, no. 13, pp. 118-123, 2019.

[32] Y. Zhou and Y. Zhang, "Optimal strategy for e-tailer issuing diamond card considering shipping policy," Computers \& Industrial Engineering, vol. 150, Article ID 106877, 2020.

[33] F. Grenouilleau, A. Legrain, N. Lahrichi, and L.-M. Rousseau, "A set partitioning heuristic for the home health care routing and scheduling problem," European Journal of Operational Research, vol. 275, no. 1, pp. 295-303, 2019.

[34] Y. Lu, Y. Xu, E. Herrera-Viedma, and Y. Han, "Consensus of large-scale group decision making in social network: the minimum cost model based on robust optimization," Information Sciences, vol. 547, pp. 910-930, 2021.

[35] S. Qu, Y. Xu, Z. Wu et al., "An interval-valued best-worst method with normal distribution for multi-criteria decisionmaking," Arabian Journal for Science and Engineering, 2020.

[36] S. Qu, Y. Han, Z. Wu, and R. Hassan, "Consensus modeling with asymmetric cost based on data-driven robust optimization," Group Decision and Negotiation, 2020.

[37] S. Qu, Y. Zhou, Y. Zhang, M. I. M. Wahab, G. Zhang, and Y. Ye, "Optimal strategy for a green supply chain considering shipping policy and default risk," Computers \& Industrial Engineering, vol. 131, pp. 172-186, 2019.

[38] Y. Ji, S. Qu, Z. Wu, and Z. Liu, "A fuzzy robust weighted approach for multi-criteria bilevel games," IEEE Transactions on Industrial Informatics, vol. 16, no. 8, pp. 5369-5376, 2020.

[39] Y. Kuo, C.-C. Wang, and P.-Y. Chuang, "Optimizing goods assignment and the vehicle routing problem with time- dependent travel speeds," Computers \& Industrial Engineering, vol. 57, no. 4, pp. 1385-1392, 2009.

[40] L. Zhang, Y. Wang, T. Fei, and H. Ren, "The research on low carbon logistics routing optimization based on DNA-ant colony algorithm," Discrete Dynamics in Nature and Society, vol. 2014, Article ID 893851, 13 pages, 2014.

[41] P. Hungerländer and C. Truden, "Efficient and easy-to-implement mixed-integer linear programs for the traveling salesperson problem with time windows," Transportation Research Procedia, vol. 30, pp. 157-166, 2018.

[42] A. L. Soyster and F. H. Murphy, "Data driven matrix uncertainty for robust linear programming," Omega, vol. 70, pp. 43-57, 2017.

[43] V. Cacchiani, J. Qi, and L. Yang, "Robust optimization models for integrated train stop planning and timetabling with passenger demand uncertainty," Transportation Research Part B: Methodological, vol. 136, pp. 1-29, 2020.

[44] M. A. M. Abdel-Aal and S. Z. Selim, "Robust optimization for selective newsvendor problem with uncertain demand," Computers \& Industrial Engineering, vol. 135, pp. 838-854, 2019.

[45] Q. Leng, S. Wang, Y. Qin, and Y. Li, "An effective method to determine whether a point is within a convex hull and its generalized convex polyhedron classifier," Information Sciences, vol. 504, pp. 435-448, 2019.

[46] S. L. Shishkin, "Optimization under non-convex Quadratic Matrix Inequality constraints with application to design of optimal sparse controller," IFAC-PapersOnLine, vol. 50, no. 1, pp. 10754-10759, 2017.

[47] D. Zeng, J. Yao, S. Yang et al., "Online risk assessment and coordinated decision scheme for emergency load shedding control," Automation of Power Systems, vol. 39, no. 20, pp. 66-71, 2015.

[48] A. Chassein, T. Dokka, and M. Goerigk, "Algorithms and uncertainty sets for data-driven robust shortest path problems," European Journal of Operational Research, vol. 274, no. 2, pp. 671-686, 2019.

[49] A. Chassein and M. Goerigk, "Minmax regret combinatorial optimization problems with ellipsoidal uncertainty sets," European Journal of Operational Research, vol. 258, no. 1, pp. 58-69, 2017.

[50] J. Guo, X. Wang, S. Fan, and M. Gen, "Forward and reverse logistics network and route planning under the environment of low-carbon emissions: a case study of Shanghai fresh food E-commerce enterprises," Computers \& Industrial Engineering, vol. 106, pp. 351-360, 2017. 